Polymer Journal, Vol. 39, No. 11, pp. 1157-1166 (2007)

(C)2007 The Society of Polymer Science, Japan

\title{
Formation of Stable Holographic Polymer Dispersed Liquid Crystal Grating with High Diffraction Efficiency Assisted by in situ Hydrolysis-Condensation of Trialkoxysilylalkyl Group of Methacrylate Component
}

\author{
Yeong Hee CHO, Go SUZUKI, and Yusuke KAWAKAMI ${ }^{\dagger}$ \\ School of Materials Science, Japan Advanced Institute of Science and Technology, 1-1 Asahidai, Nomi 923-1292, Japan
}

(Received June 25, 2007; Accepted August 7, 2007; Published September 26, 2007)

\begin{abstract}
Stable transmission holographic polymer dispersed liquid crystal grating with reasonably high diffraction efficiency (72\%, induction period $576 \mathrm{~s}$ ) could be prepared via radical network formation of trimethylolpropane triacrylate (only $10 \mathrm{wt} \%$, photo-cross-linkable, further diluted with $10 \mathrm{wt} \%$ 1-vinyl-2-pyrrolidone) and 3-methacryloxypropyltrimethoxysilane ( $80 \mathrm{wt} \%$, non cross-linkable by radical polymerization, but cross-linkable by hydrolysiscondensation) in the presence of $35 \mathrm{wt} \%$ TL 203 as a liquid crystal, assisted by simultaneous hydrolysis-condensation of trimethoxysilyl group. The hydrolysis-condensation was induced by moisture in reagents and proton species produced from 3, 3'-carbonylbis[7'-diethylaminocoumarine] as a photo-sensitizer and diphenyliodonium hexafluorophosphate as a photo-initiator. Grating could not be formed for the same system with 3-methacryloxypropyltrimethylsilane (non hydrolyzable and not cross-linkable).

Rather decreased efficiency of 3-methacryloxypropyltriethoxysilane system (13\% efficiency, $693 \mathrm{~s}$ induction period) was remarkably improved to $75 \%$ ( $75 \mathrm{~s}$ induction period) by introducing hydrophilic urethane group into the spacer to facilitate easier hydrolysis. The long grating spacing of $0.9 \mu \mathrm{m}$ indicates very small volume shrinkage. The maximum diffraction efficiency (85\%) was observed at 60-70 wt \% 3- $N$-(2-methacryloxyethoxycarbonyl)aminopropyltriethoxysilane, but with a little larger volume shrinkage.

Introduction of aminoalcohol group in the spacer could further shorten the induction period to $18 \mathrm{~s}$, but the diffraction efficiency was rather low (20\%). [doi:10.1295/polymj.PJ2007086]

KEY WORDS Trialkoxysilylalkyl Methacrylate / Hydrolysis-condensation Reaction / Diffraction Efficiency / Holographic Polymer Dispersed Liquid Crystal / Phase Separation / Siloxane Network Formation / Volume Shrinkage /
\end{abstract}

In recent years, holographic polymer dispersed liquid crystal (HPDLC) systems has attracted much interest due to their unique switching property by electric field to make them applicable to information displays, optical shutters, and information storage media. ${ }^{1-10}$ Photo-polymerizable materials, typically multi-functional acrylates, have been mostly studied as materials for HPDLC because of their advantages of large refractive index modulation, low cost, and easy fabrication and modification. ${ }^{11-17}$ However, these HPDLC materials still have significant drawbacks such as volume shrinkage, low reliability, and poor long term stability.

Since the performance of HPDLC is governed by the stability of precisely formed periodic polymer rich layer and LC rich layer created by interference of two incident laser beams, control of the formation of polymer matrix and phase separation of LC are very important. ${ }^{18-24}$ The principal role of multi-functional acrylate in grating formation is to make the LC phase-separate by the formation of cross-linked polymer matrix. Control of the rate and density of crosslinking in polymer matrix is one of the most important factors, which determines the size, distribution, and shape of phase-separated LC domains, accordingly the final morphology and performance. In order to obtain clear phase separation of LC from polymer matrix to homogeneous droplets, high cross linking density, but not rapid cross-linking, is very important. Too rapid initial cross-linking by multi-functional acrylate makes it difficult to control the diffusion and phase separation of LC. At the same time, high ultimate conversion of polymerizable double bond leading to high cross-linking is important for long-term stability. These are not easy to achieve at the same time.

Till now optimization of cross-linking process has been mainly pursued by controlling the average functionality of multi-functional acrylate by mixing dipentaerythritol pentaacrylate (DPEPA), trimethylolpropane triacrylate (TMPTA) and tri(propyleneglycol) diacrylate, or by diluting the system with mono-functional vinyl compound like 1-vinyl-2-pyrollidone (NVP). ${ }^{25-28}$ In case of TMPTA, considerably high concentration was used. Mono-functional NVP adjusts the initial polymerization rate and final conversion of acrylate functional groups by lowering the concentration of cross-linkable double bonds. ${ }^{29}$ However, the

${ }^{\dagger}$ To whom correspondence should be addressed (Tel: +81-761-51-1630, Fax: +81-761-51-1635, E-mail: kawakami@jaist.ac.jp). 
effects were so far limited, and these systems still caused serious volume shrinkage and low final conversion of polymerizable groups. Thus, the gratings are not long-term stable, either. Moreover, the phase separation of LC component during the matrix formation was governed only by its intrinsic property difference against polymer matrix, accordingly not wellcontrolled. These systems could be called as "passive grating formation" systems.

Recently, we have concentrated on the organic-inorganic hybrid materials ${ }^{30-36}$ to improve the drawbacks of HPDLC system by the incorporation of siloxane components in the grating formation with the intention to assist the efficient phase separation of monomer and LC components with controlled manner by taking advantages of highly incompatible nature of siloxane component against polymer matrix and its flexibility to ease the diffusion of LC. ${ }^{37-43}$ Introduction of polyhedral oligosilsesquioxane structure also improved the performance of the formed grating. ${ }^{44}$ Thus, if we consider the structure and reactivity of siloxane compounds in relation with the property, it will be possible to propose new systems to improve the performance of HPDLC gratings.

Our idea is to improve the property of gratings through importing the siloxane network formation in polymer matrix, by not only lowering the contribution of initial rapid radical cross-linking of TMPTA and realizing complete conversion of double bonds, but also maintaining the desirable total cross-linking density assisted by hydrolysis-condensation cross-linking of trialkoxysilyl group in the methacrylate component to control the phase separation of LC from polymer matrix. ${ }^{45}$ Such cross-linking can be promoted by the proton species produced from the initiating system together with radical species by photo-reaction. ${ }^{46-48}$ In our system, phase separation of LC is not so fast compared with simple multi-functional acrylate system, and secondary cross-linking by the formation of siloxane network enforce the LC to completely phase-separate to homogeneous droplets, and high diffraction efficiency could be expected. We named this process as proton "assisted grating formation."

These systems should provide many advantages over traditional systems induced only by radical polymerization by improving: 1) the volume shrinkage by reducing the contribution of radical initial cross-linking by importing the siloxane network in whole polymer networks, 2) the contrast of siloxane network

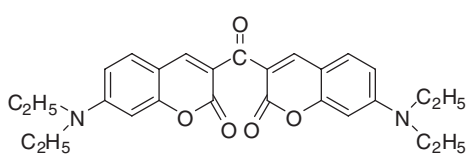

3,3'-Carbonylbis(7-diethylaminocoumarin)(KC) formed by the hydrolysis of $\omega$-methacryloxyalkyltrialkoxysilane against polymer matrix, and 3) the stability of final gratings via combination of the characteristics of siloxane gel and rather loosely cross-linked radically polymerized system.

Special emphasis was given to systematical study of the effectiveness of 3-methacryloxypropyltriethoxysilane with hydrophilic urethane group in spacer on performance of gratings. Progress of cross-linking was measured by photo-DSC, and correlated to realtime diffraction efficiency.

\section{EXPERIMENTAL}

\section{Materials for recording solution}

Multi-functional acrylate, trimethylolpropane triacrylate (TMPTA), purchased from Aldrich Chemical Co., was used as radically cross-linkable monomer to tune the reaction rate and cross-linking density. Methacryloxymethyltrimethxysilane (MM-TMS), methacryloxymethyltrimethoxysilane (MM-TMOS), 3methacryloxypropyltrimethoxysilane (MP-TMOS), 3-methacryloxypropyltriethoxysilane (MP-TEOS), 3$\mathrm{N}$-(2 - methacryloxyethoxycarbonyl)aminopropyltriethoxysilane (MU-TEOS), and 3- $N$-(3-methacryloxy2-hydroxypropyl)aminopropyltriethoxysilane (MHTEOS), purchased from Gelest, Inc., were used as reactive diluents. Methacrylate with trialkoxysilane are capable of not only radical polymerization but also hydrolysis-condensation. The structure of methacrylates used in this study are shown in Figure 1. 1-Vinyl-2pyrrolidone (NVP) was used as another radically polymerizable reactive diluent. A commercial nematic LC, TL203 $\left(\mathrm{T}_{\mathrm{NI}}=74.6^{\circ} \mathrm{C}, \mathrm{n}_{\mathrm{e}}=1.7299, \mathrm{n}_{\mathrm{o}}=1.5286\right.$, $\Delta \mathrm{n}=0.2013, \Delta \varepsilon=11.0$ ), purchased from Merck \& Co. Inc., was used without any purification.

\section{Composition of Photo-initiator System and Recording Solution}

Photo-sensitizer (PS) and photo-initiator (PI) having sensitivity to visible wavelength of Nd-YAG laser $(\lambda=532 \mathrm{~nm})$ selected for this study are $3,3^{\prime}$-carbonylbis(7-diethylaminocoumarin) (KC, Kodak) and diphenyliodonium hexafluorophosphate (DPI, AVOCADO research chemicals Ltd.), respectively, which produce both cationic and radical species. ${ }^{49-51}$ The concentrations of the PS and PI were used in the range of 0.2 and $2.0 \mathrm{wt} \%$ to matrix components, respectively.

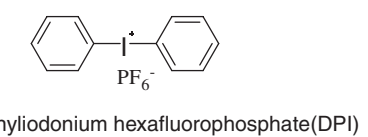




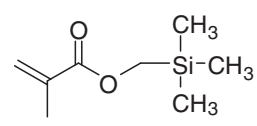

Methacryloxymethyltrimethylsilane (MM-TMS)

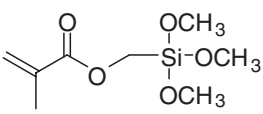

Methacryloxymethyltrimethoxysilane (MM-TMOS)

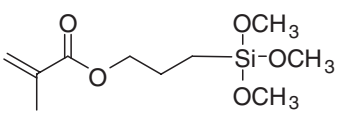

3-Methacryloxypropyltrimethoxysilane (MP-TMOS)

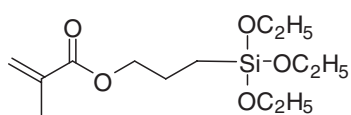

3-Methacryloxypropyltriethoxysilane (MP-TEOS)

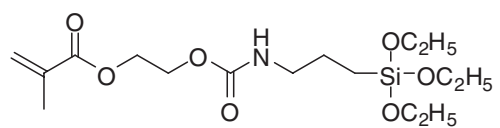

3-N-(2-methacryloxyethoxycarbonyl)aminopropyltriethoxysilane (Mu-TEOS)

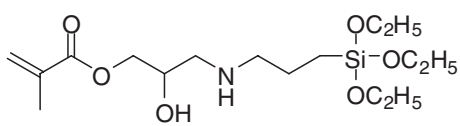

3-N-(3-methacryloxy-2-hydroxypropyl)aminopropyltriethoxysilane (MH-TEOS)

Figure 1. Structures of $\omega$-methacryloxyalkyltri-alkyl or -alkoxysilanes.

The ratio of polymer matrix components, a fast curing TMPTA and $\omega$-methacryloxyalkyltrialkoxysilane, was changed from $80: 10$ to $10: 80 \mathrm{wt} \%$ in the relative ratio with $10 \mathrm{wt} \%$ reactive diluent NVP. Recording solution was prepared by mixing the matrix components $(65 \mathrm{wt} \%)$ and TL203 (35 wt \%), and injected into a glass cell with a gap of $20 \mu \mathrm{m}$ controlled by bead spacer.

\section{Measurement of photo-DSC and FT/IR}

The rate of polymerization was estimated from the heat flux monitored by photo-differential scanning calorimeter (photo-DSC) equipped with a dual beam laser light of $532 \mathrm{~nm}$ wavelength. Matrix compounds were placed in uncovered aluminum DSC pans and cured with laser light by keeping the isothermal state of $30^{\circ} \mathrm{C}$ at various light intensities.

Infrared absorption spectra in the range 4000$400 \mathrm{~cm}^{-1}$ were recorded on polymer matrix compounds by Fourier Transform Infrared Spectroscopy (FT/IR) (Perkin-Elmer, Spectrum One).

\section{Optical Setup for Transmission Holographic Gratings}

Nd:YAG solid-state continuous wave laser with $532 \mathrm{~nm}$ wavelength (Coherent Inc., Verdi-V2) was used as the irradiation source as shown in Figure 2.

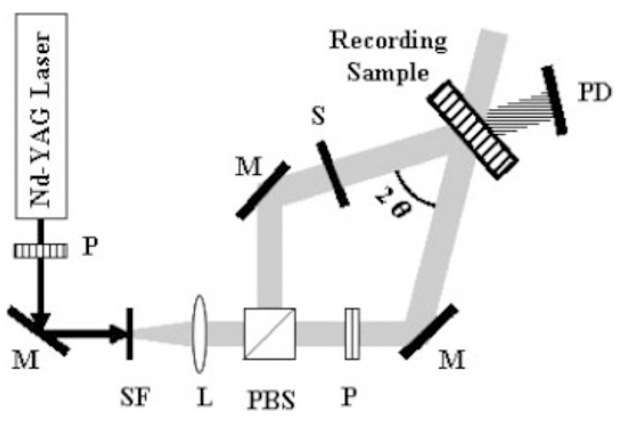

Figure 2. Experimental setup for the holographic recording and real-time reading; P: $1 / 2 \lambda$ plate, M: mirror, SF: spatial filter, L: collimating lens, PBS: polarizing beam splitter, S: shutter, $2 \theta$ : external inter-beam angle, PD: power detector.

The beam was expanded and filtered by spatial filters, and collimated by collimator lens. $s$-Polarized beams were generated and split by controlling the two $\lambda / 2$ plates and polarizing beam splitter. Thus separated two $s$-polarized beams with equal intensities were reflected by two mirrors and irradiated to recording solution at a pre-determined external beam angle $(2 \theta)$ which was controlled by rotating the motordriven two mirrors and moving the rotation stage along the linear stage. In this research, the external incident beam angle was fixed at $16^{\circ}(\theta)$ against the line perpendicular to the plane of the recording cell. Realtime diffraction efficiency was measured by monitoring the intensity of diffracted beam when the shutter was closed at a constant time interval during the hologram recording. After the hologram was recorded, diffraction efficiency was measured by rotating the hologram precisely by constant angle by using motor-driven controller, with the shutter closed to cut-off the reference light, to determine the angular selectivity. Holographic gratings were fabricated at $20 \mathrm{~mW} / \mathrm{cm}^{2}$ intensity for one beam, and the optimum condition was established to obtain the high diffraction efficiency, high resolution, and excellent long-term stability after recording. Diffraction efficiency is defined as the ratio of diffraction intensity after recording to transmitting beam intensity before recording.

\section{Morphology of Holographic Gratings}

Surface morphology of gratings was examined with scanning electron microscope (SEM, HITACHI, S4100). The samples for measurement were prepared by freeze-fracturing in liquid nitrogen, and washed with methanol for $24 \mathrm{~h}$ to extract the TL203, in case necessary. Exposed surface of the samples for SEM was coated with a very thin layer of Pt-Pd to minimize artifacts associated with sample charging (HITACHI, E-1030 ion sputter). 


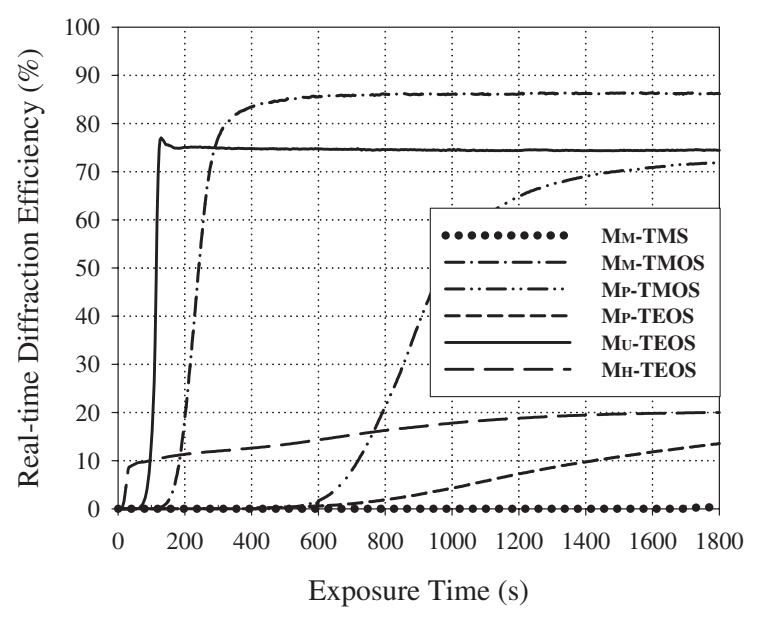

Figure 3. Real-time diffraction efficiency of the gratings formed with various $\omega$-methacryloxyalkyltrialkoxysilanes in the recording solution with $65 \mathrm{wt} \%$ matrix compounds of TMPTA: $\omega$-methacryloxyalkyltrialkoxysilane:NVP $=10: 80: 10 \mathrm{wt} \%$ and $35 \mathrm{wt} \%$ TL203, and KC-DPI $(0.2 \mathrm{wt} \%-2 \mathrm{wt} \%$ to matrix compounds) with one beam intensity of $20 \mathrm{~mW} / \mathrm{cm}^{2}$.

\section{RESULTS AND DISCUSSION}

\section{Effectiveness of MM-TMOS on Formation of Holo- graphic Gratings}

As a preliminary experiment, MM-TMS and MMTMOS were compared as a diluent for the polymer matrix component (totally $65 \mathrm{wt} \%$, TMPTA:MMTMS, or MM-TMOS:NVP $=10: 80: 10$ in wt $\%$, average double bond functionality $=1.1$ on mole base), together with $35 \mathrm{wt} \%$ LC of TL203. As shown in Figure 3 gratings could not be formed with MMTMS even with $30 \mathrm{~min}$ irradiation of light, because of the low average functionality of the polymerization system. G. P. Crawford reported that HPDLC gratings made with monomer mixtures with average double bond functionality less than 1.3 were mechanically very weak. ${ }^{52}$ In general, it is difficult to form holographic gratings with low concentration of multifunctional acrylate (average double bond functionality $<1.2$ ) by dilution with mono-functional component in radical polymerization.

Dramatic enhancing in the diffraction efficiency to about $86 \%$ (induction period of $144 \mathrm{~s}$ ) was observed in case of MM-TMOS, even with only $10 \mathrm{wt} \%$ TMPTA by using $0.2 \mathrm{wt} \% \mathbf{K C}$ and $2 \mathrm{wt} \%$ DPI. Only trimethoxysilyl and trimethylsilyl parts are different in these two formulations. Hydrolysis of trimethoxysilyl group by moisture and following condensation seems responsible for the increased diffraction efficiency.

Effects of Alkyl and Spacer Groups in $\omega$-Methacryloxyalkyltrialkoxysilanes on the Formation and Performance of Gratings

In order to systematically study the influence of alkyl group and spacer group of $\omega$-methacryloxyalkyltrialkoxysilanes on the formation and performance of the formed gratings, their chemical structures were modified as shown in Figure 1. The relative concentration was set as TMPTA: $\omega$-methacryloxyalkyltrialkoxysilane:NVP $=10: 80: 10 \mathrm{wt} \%$ to clearly extract the effects of hydrolysis-condensation of trialkoxysilyl group on the formation of the gratings and the performance of the formed gratings.

Figure 3 shows the real-time diffraction efficiency of holographic gratings formed with various $\omega$-methacryloxyalkyltrialkoxysilanes capable of radical photo-polymerization and hydrolysis-condensation.

When spacer was changed from methylene to propylene (MP-TMOS), the diffraction efficiency was dropped to $72 \%$ with longer induction period (576 s). This seems to be because of the higher hydrophobicity of the spacer group compared with MMTMOS. The rate of the hydrolysis-condensation of trialkoxysilyl functions seems very important.

By changing the trialkoxysilyl functional group from trimethoxy to triethoxy (MP-TMOS to MPTEOS) with the same propylene spacer, not only the diffraction efficiency was decreased to $13 \%$, but the induction period was also elongated to $693 \mathrm{~s}$, which again strongly suggested that the hydrolysiscondensation process of trialkoxysilane function is playing an essential role in grating formation.

In grating formation, induction period basically depends on the time of the formation of cross-linked polymer matrix. In classical grating formation by radical polymerization of multi-functional acrylates, induction period is observed because polymerization does not start until the complete consumption of oxygen present in the system as an inhibitor. In the present system, the induction period depends on the actual gelation time of recording solution assisted by hydrolysis-condensation of trialkoxysilyl functions. The induction period varies by the physical property of $\omega$-methacryloxyalkyltrialkoxysilane derivatives. The rate of the hydrolysis-condensation of trialkoxysilyl functions by moisture strongly depends on the hydrophobicity of the methacrylate monomer. Polymerization of recording solution leads to changes in the chemical potential of the system, and increases the miscibility gap between LC and polymerized matrix.

To investigate the effects of chemical structures of spacer between trialkoxysilylalkyl group and methacrylate group, hydrophilic urethane and hydroxylpropylene groups were introduced in the spacer of the monomer structure. The highest diffraction efficiency of $75 \%$ and remarkably shorter induction period of $75 \mathrm{~s}$ were obtained for grating formed with MU-TEOS having urethane linkage in spacer group. In addition, gratings formed with MH-TEOS having hydroxylpro- 
pylene group in the spacer showed the shortest induction period of $18 \mathrm{~s}$, although the diffraction efficiency was considerably low (20\%).

\section{Analysis of Grating Formation Process by Photo-DSC and FT/IR Spectra}

Photo-polymerization rate profiles and conversions of reactive monomers were estimated from the heat flux measured by the photo-DSC. When the lowest laser intensity of $2 \mathrm{~mW} / \mathrm{cm}^{2}$ was used, the photoDSC apparently showed three major peaks as shown in Figure 4.

The enthalpy of polymerization could be roughly estimated as 50,000 and $20,000 \mathrm{~J} / \mathrm{mol}$, respectively, for double bond opening and silanol condensation. ${ }^{53}$

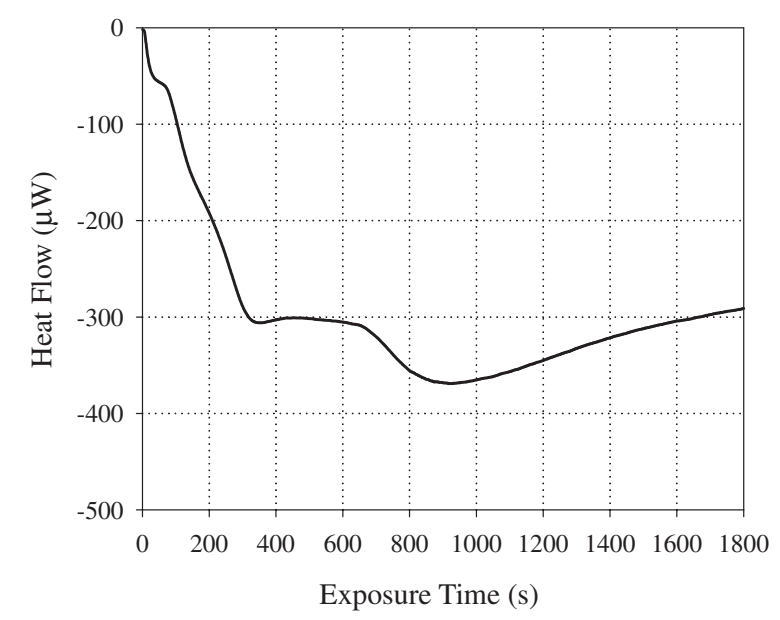

Figure 4. Heat flow of photo-DSC of matrix compounds with MU-TEOS at the ratio of TMPTA:MU-TEOS:NVP = 10:80:10 wt \%, and KC-DPI $(0.2 \mathrm{wt} \%-2 \mathrm{wt} \%$ to matrix compounds), with laser intensity of $2 \mathrm{~mW} / \mathrm{cm}^{2}$.
Although it is not easy to assign which process is contributing the most to each peak, it will be reasonable to assign the first peak to the initial radical polymerization and cross-linking of TMPTA with four functional group, and second and third for hydrolysis-condensation and polymerization of methacrylate, or vice versa. It might be possible to estimate the reaction rate by monitoring the rate of heat evolution from the polymerization sample.

IR analysis indicated the disappearance of carboncarbon double bond in accordance with the heat evolution as shown in Figure 5.

The sharp absorption peak at $1634 \mathrm{~cm}^{-1}$ corresponds to carbon-carbon double bond gradually decreased with increasing the irradiation time even with the irradiation intensity of $2 \mathrm{~mW} / \mathrm{cm}^{2}$, and disappeared completely by irradiation of $113 \mathrm{~mW} / \mathrm{cm}^{2}$ laser intensity for $300 \mathrm{~s}$. The absorption peak at $879 \mathrm{~cm}^{-1}$ corresponds to $\mathrm{Si}-\mathrm{O}-\mathrm{Si}$ bending bands was observed from $480 \mathrm{~s}$ irradiation time as shown in Figure 5(d). The peak became stronger by increasing the irradiation time or by irradiation with higher laser intensity of $113 \mathrm{~mW} / \mathrm{cm}^{2}$. These results are very well in accordance with our suggestion of reaction profile.

Since the polymerization of TMPTA proceeds very rapidly with heat evolution, care must be taken to ensure that the reaction rate does not exceed the time resolution of the instrument, as the DSC response time is of the order of $0.5 \mathrm{~s}$. Relatively low light intensity of $8 \mathrm{~mW} / \mathrm{cm}^{2}$ was used to increase the induction time for the grating formation as shown in Figure 6. Actual heat evolution in the actual grating formation with $40 \mathrm{~mW} / \mathrm{cm}^{2}$ of two beam intensity should be much faster than the present result.

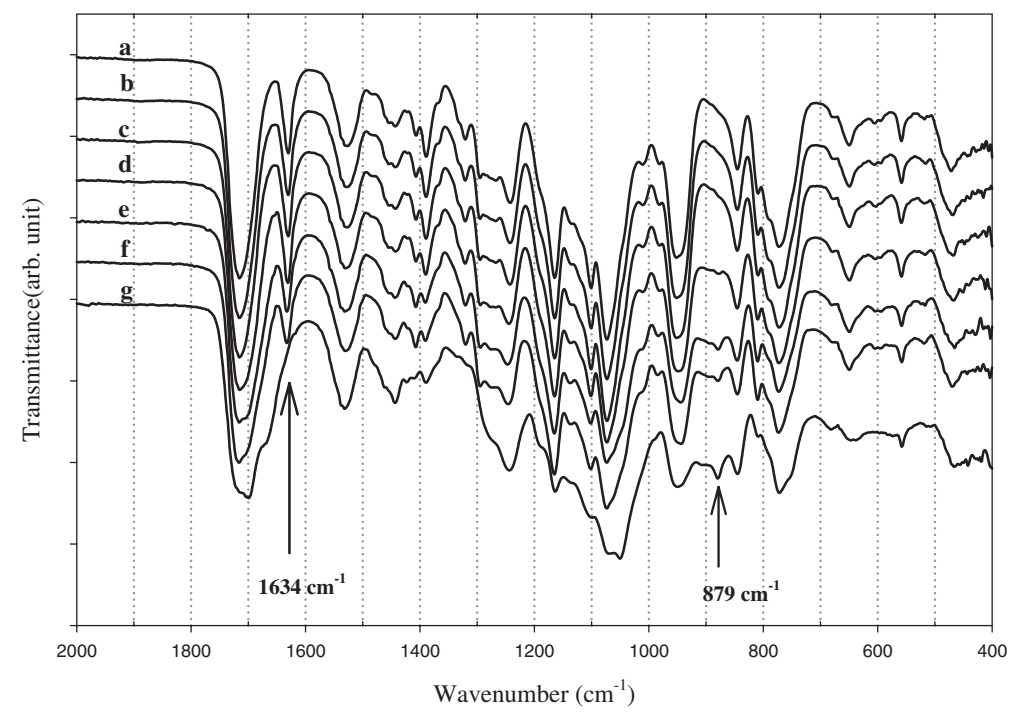

Figure 5. FT/IR spectra of matrix compounds with MU-TEOS at the ratio of TMPTA:MU-TEOS:NVP $=10: 80: 10 \mathrm{wt} \%$, and KCDPI (0.2 wt \%-2 wt \% to matrix compounds) according to the irradiation time with laser intensity of $2 \mathrm{~mW} / \mathrm{cm}^{2}$ : (a) before irradiation, (b) $60 \mathrm{~s}$, (c) $180 \mathrm{~s}$, (d) $480 \mathrm{~s}$, (e) $2000 \mathrm{~s}$, (f) $1800 \mathrm{~s}$, and (b) $300 \mathrm{~s}$ irradiation with laser intensity of $113 \mathrm{~mW} / \mathrm{cm}^{2}$. 


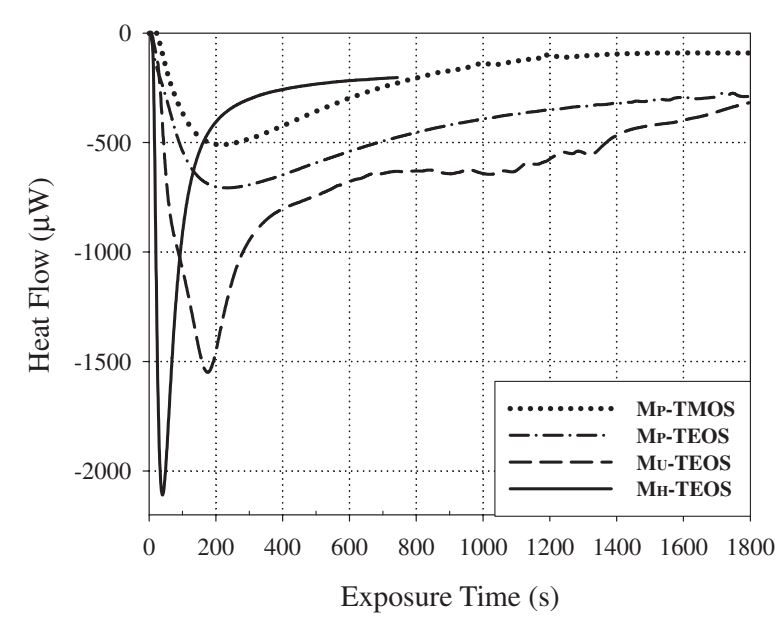

Figure 6. Photo-DSC analysis of matrix compounds with various $\omega$-methacryloxyalkyltrialkoxysilanes at the ratio of TMPTA: $\omega$-methacryloxyalkyltrialkoxysilane:NVP $=10: 80: 10$ wt $\%$ and KC-DPI $(0.2 \mathrm{wt} \%-2 \mathrm{wt} \%$ to matrix compounds), with laser intensity of $8 \mathrm{~mW} / \mathrm{cm}^{2}$.

By comparing Figures 3 and 6, we can notice that the grating formation (total beam intensity $=40$ $\mathrm{mW} / \mathrm{cm}^{2}$ ) of the system is slower than the appearance of the peak of heat evolution. This indicates that the gratings are formed after some cross-linking was formed. In case of propylene spacer, grating formation seems much slower than heat evolution maximum. This strongly suggests that the hydrolysis-condensation cross-linking after the initial radical polymerization is mainly contributing to the grating formation for these monomers.

Contrary to this, in the case of the methacrylate with urethane or hydroxypropylene spacer, the time difference between the heat evolution and grating formation became much shorter, and the profile of the heat evolution became sharp. MU-TEOS had the highest reaction rate and the sharpest heat evolution profile. This may indicate that cross-linking by both radical polymerization and hydrolysis condensation is occurring almost simultaneously, in this case. These behaviors directly influenced the diffraction efficiency as described in Figure 3. If the cross-linking formation by the polymerization of multi-functional acrylate, or by hydrolysis condensation is too fast, polymer matrix will form highly cross-linked network in the initial stage, which restricts the diffusion of TL203 to low intensity regions of interference fringes, and results in low diffraction efficiency. Monomer with urethane spacer seems to have optimum balance of the initial cross-linking and diffusion of LC in grating formation among the samples studied, and resulted in the highest diffraction efficiency with moderate induction time.

From these results, we can point out the important role of the function of cross linking by hydrolysis con-

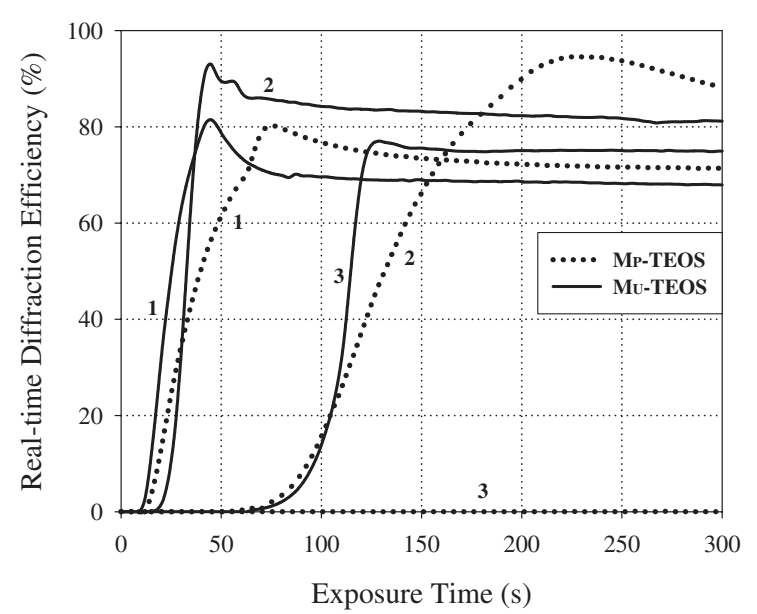

Figure 7. Real-time diffraction efficiency of the gratings formed with MP-TEOS and MU-TEOS in recording solution of 65 wt $\%$ matrix compounds of TMPTA: $\omega$-methacryloxyalkyltriethoxysilane:NVP $=90-\mathrm{X}: \mathrm{X}: 10 \mathrm{wt} \%), 35 \mathrm{wt} \% \quad$ TL203, and KC-DPI ( $0.2 \mathrm{wt} \%-2 \mathrm{wt} \%$ to matrix compounds), with one beam intensity of $20 \mathrm{~mW} / \mathrm{cm}^{2}: 1,2$, and 3 represent the $\mathrm{X}$ is $10 \mathrm{wt} \%$, $50 \mathrm{wt} \%$, and $80 \mathrm{wt} \%$, respectively.

densation of trialkoxysilyl group on the performance of gratings, especially hydrophilic moiety of urethane or hydroxy group affected strongly on the rates of formation of cross-linking with siloxane network, even though the reaction rate was slower than the initial cross-linking by multi-functional acrylate. Modification of the formulation of recording solution with MH-TEOS might give gratings with higher diffraction efficiency, which is under study now.

To further confirm the effectiveness of urethane moiety in spacer, the concentration of MP-TEOS and MU-TEOS were varied as shown in Figure 7.

At low concentration of MP-TEOS and MU-TEOS (10 wt \%), namely with high concentration of TMPTA (80 wt \%), where TMPTA is playing an essential role in cross-linking, similarly high initial transient diffraction efficiency of about $70 \%$ and short induction periods about $12 \mathrm{~s}$ were observed for both systems due to the non-equilibrium initial cross-liking of TMPTA. When the concentration of the monomers was $50 \mathrm{wt} \%$, the grating with remarkably higher diffraction efficiency was formed much faster with MU-TEOS than with MP-TEOS. By increasing the concentration of MU-TEOS and MP-TEOS to $80 \mathrm{wt} \%$, their differences in diffraction efficiency and induction periods became more remarkable. The MPTEOS system could not form any grating in $300 \mathrm{~s}$, although it could start to form grating after $700 \mathrm{~s}$.

From these results, the chemical structure of spacer of $\omega$-methacryloxyalkyltriethoxysilane seems to play an essential role to assist the formation of cross-linking networks in polymer matrix. The siloxane network formation and radical polymerization of MU-TEOS 


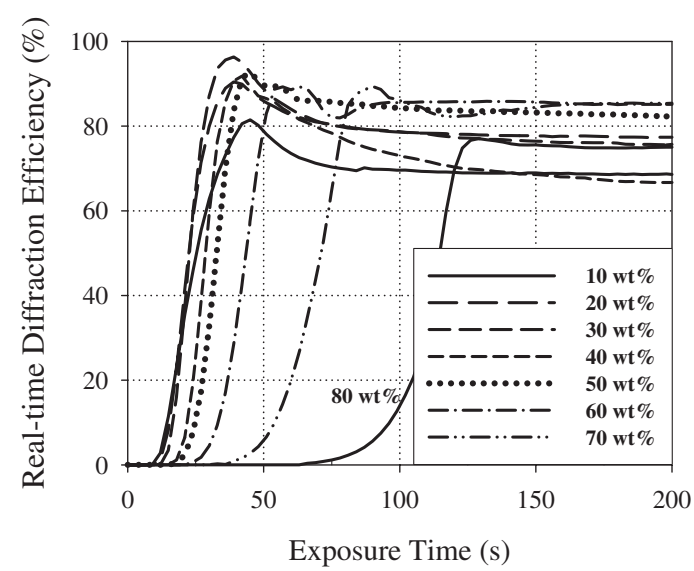

Figure 8. Real-time diffraction efficiency of the gratings formed with MU-TEOS in recording solution with $65 \mathrm{wt} \%$ matrix compounds of TMPTA:MU-TEOS:NVP $=90-\mathrm{X}: \mathrm{X}: 10 \mathrm{wt} \%$ ( $\mathrm{X}$ is from 10 to 80 ), $35 \mathrm{wt} \%$ TL203, and KC-DPI (0.2 wt \%$2 \mathrm{wt} \%$ to matrix compounds), at the one beam intensity of $20 \mathrm{~mW} / \mathrm{cm}^{2}$.

might be simultaneously promoted by hydrolysis of triethoxysilyl group and condensation of silanol assisted by the hydrophilic urethane group.

\section{Control of Cross-linking Density and Reaction Rates} by Varying the Concentration of TMPTA and MU-TEOS

Relative concentration of TMPTA and MU-TEOS was further varied to control the cross-linking density and the rate of grating formation in matrix components (totally $65 \mathrm{wt} \%$ matrix components) as TMPTA:MU-TEOS:NVP $=90-\mathrm{X}: \mathrm{X}: 10 \mathrm{wt} \% \quad(\mathrm{X}$ is from 10 to 80 ), $35 \mathrm{wt} \%$ TL203, and KC-DPI $(0.2 \mathrm{wt} \%-2 \mathrm{wt} \%$ to matrix compounds) as shown in Figure 8 .

At lower concentration of MU-TEOS, cross-linking of TMPTA by initial radical mechanism gave high transient diffraction efficiency. With increasing the concentration of MU-TEOS, or decreasing the concentration of TMPTA, diffraction efficiencies at 200 s exposure time were similarly high over $70 \%$, although the induction periods for grating formation were prolonged. Diffraction efficiency with $80 \mathrm{wt} \%$ MU-TEOS was about 75\%, and stable. Longer induction periods for the higher concentration of MUTEOS, however, clearly indicate that the primary cross-linking was formed by radical polymerization of TMPTA, and the siloxane cross-linking was the secondary. However, introduction of urethane group in the spacer makes the hydrolysis condensation relatively faster than the monomer without urethane group. This is an important role of urethane group.

Figure 9 shows the photo-DSC of the gratings formed with MU-TEOS in recording solution of

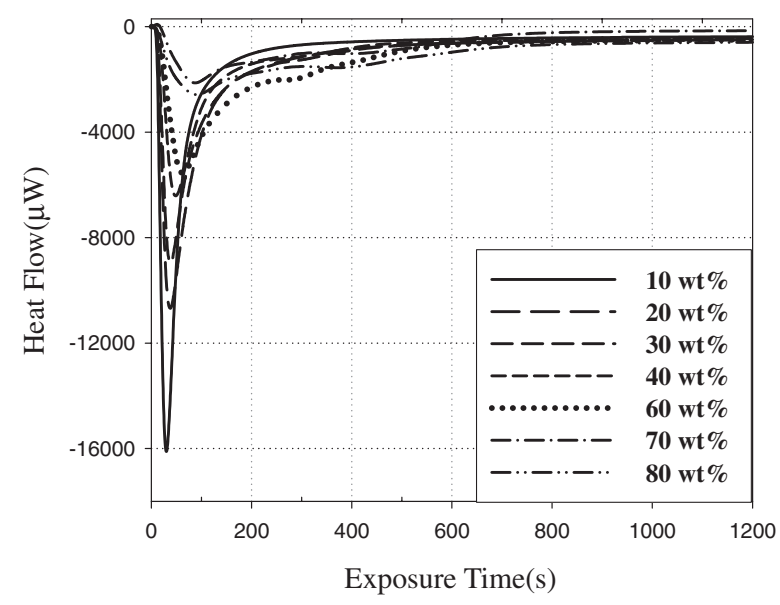

Figure 9. Photo-DSC analysis of the gratings formed with MU-TEOS in recording solution with $65 \mathrm{wt} \%$ matrix compounds of TMPTA:MU-TEOS:NVP $=90-\mathrm{X}: \mathrm{X}: 10 \mathrm{wt} \%$ (X is from 10 to 80$), 35 \mathrm{wt} \%$ TL203, and KC-DPI $(0.2 \mathrm{wt} \%-2 \mathrm{wt} \%$ to matrix compounds) at $8 \mathrm{~mW} / \mathrm{cm}^{2}$ laser intensity.

$65 \mathrm{wt} \%$ matrix compounds of TMPTA:MU-TEOS: $\mathbf{N V P}=90-\mathrm{X}: \mathrm{X}: 10 \mathrm{wt} \%$ ( $\mathrm{X}$ is from 10 to 80 ), $35 \mathrm{wt} \%$ TL203, and KC-DPI $(0.2 \mathrm{wt} \%-2 \mathrm{wt} \%$ to matrix compounds) at the laser intensity of $8 \mathrm{~mW} /$ $\mathrm{cm}^{2}$.

With increasing the concentration of MU-TEOS, the maximum heat flux shifted to longer time, and the total heat evolved was decreased, as expected. This order of reaction rates is in good agreement with the order of induction periods shown in Figure 8 suggesting that, as mentioned above, when the concentration of TMPTA is increased, reaction of TMPTA proceeds preferentially in the initial stage due to its high reactivity.

To understand the behaviors of reaction rate profile in the mixtures of TMPTA and MU-TEOS by photoDSC analysis, light intensity was varied using formulation of $65 \mathrm{wt} \%$ matrix components keeping the ratio TMPTA:MU-TEOS:NVP being 10:80:10 wt \%, with $35 \mathrm{wt} \%$ TL203, and KC-DPI $(0.2 \mathrm{wt} \%-2 \mathrm{wt} \%$ to matrix compounds) as shown in Figure 10.

As light intensity increased, the exothermic heat flow reached its maximum much rapidly. For example, at $8 \mathrm{~mW} / \mathrm{cm}^{2}$ the heat flux reached the maximum at about $180 \mathrm{~s}$, while for intensity of $152 \mathrm{~mW} / \mathrm{cm}^{2}$ at $78 \mathrm{~s}$. Furthermore, this increase in reaction rate was found to result in higher final conversions as determined from the total heat of reaction.

To summarize the results, we may consider that the radically mono-functionally polymerizable 3-methacryloxypropyltrialkoxysilane became apparently multi-functional cross-linkable monomer by hydrolysis and condensation of trialkoxysilyl group as shown in Scheme 1, which induced the high concentration of cross-linking with moderate rate by the hydrolysis. 


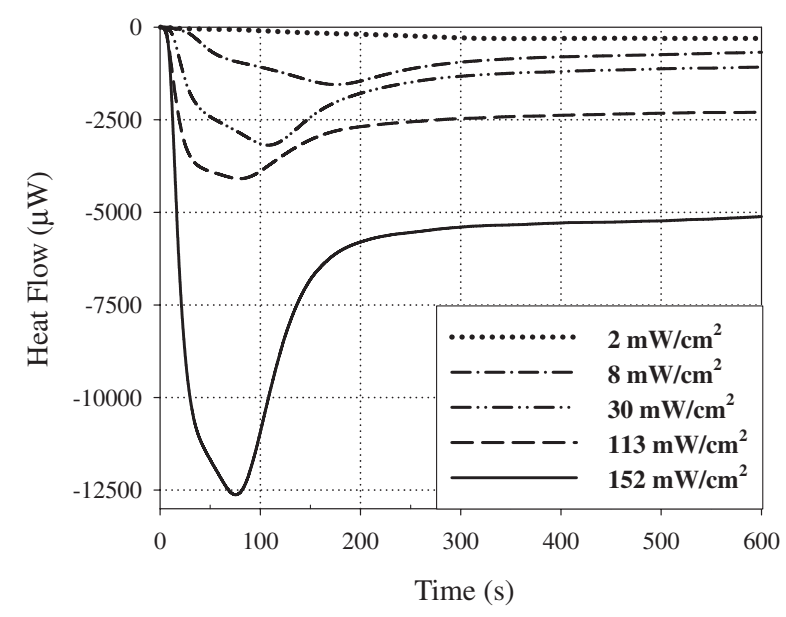

Figure 10. Photo-DSC analysis of gratings formed with MUTEOS in recording solution with $65 \mathrm{wt} \%$ matrix compounds of TMPTA:MU-TEOS:NVP is 10:80:10 wt \%, $35 \mathrm{wt} \%$ TL203, and KC-DPI ( $0.2 \mathrm{wt} \%-2 \mathrm{wt} \%$ to matrix compounds) at the various laser intensities.

In case of methacryloxymethyltrimethylsilane, cross-linking density is not high enough to form grating. This process corresponds to type 1) in Scheme 1.
In TMOS or TEOS system, the hydrolysis of trialkoxysilyl group is relatively slow compared with the fast radical polymerization of TMPTA. Thus, grating formation is not rapid, but following cross-linking by hydrolysis assisted the formation of polymer matrix and further diffusion of LC to form gratings with high diffraction efficiency. By the introduction of urethane function in the spacer, the hydrophilic nature of the spacer increases the hydrolysis of triethoxysilyl group by moisture, and converts mono-functional methacrylate to apparently multi-fumctional methacrylate, and assisted the formation of polymer matrix by radical polymerization together with cross-linking by hydrolysis condensation. This process corresponds to type 2) in Scheme 1. In case of the introduction of hydroxypropylene spacer, too much hydrophilic nature of the spacer strongly enhanced the hydrolysis of the trialkoxysilyl group, and created the situation where apparently high concentration of multi-functional (meth)acrylates in the initial polymerization solution, and resulted in rapid formation of grating by radical cross-linking, but low diffraction efficiency. This process corresponds to type 3) in Scheme 1.

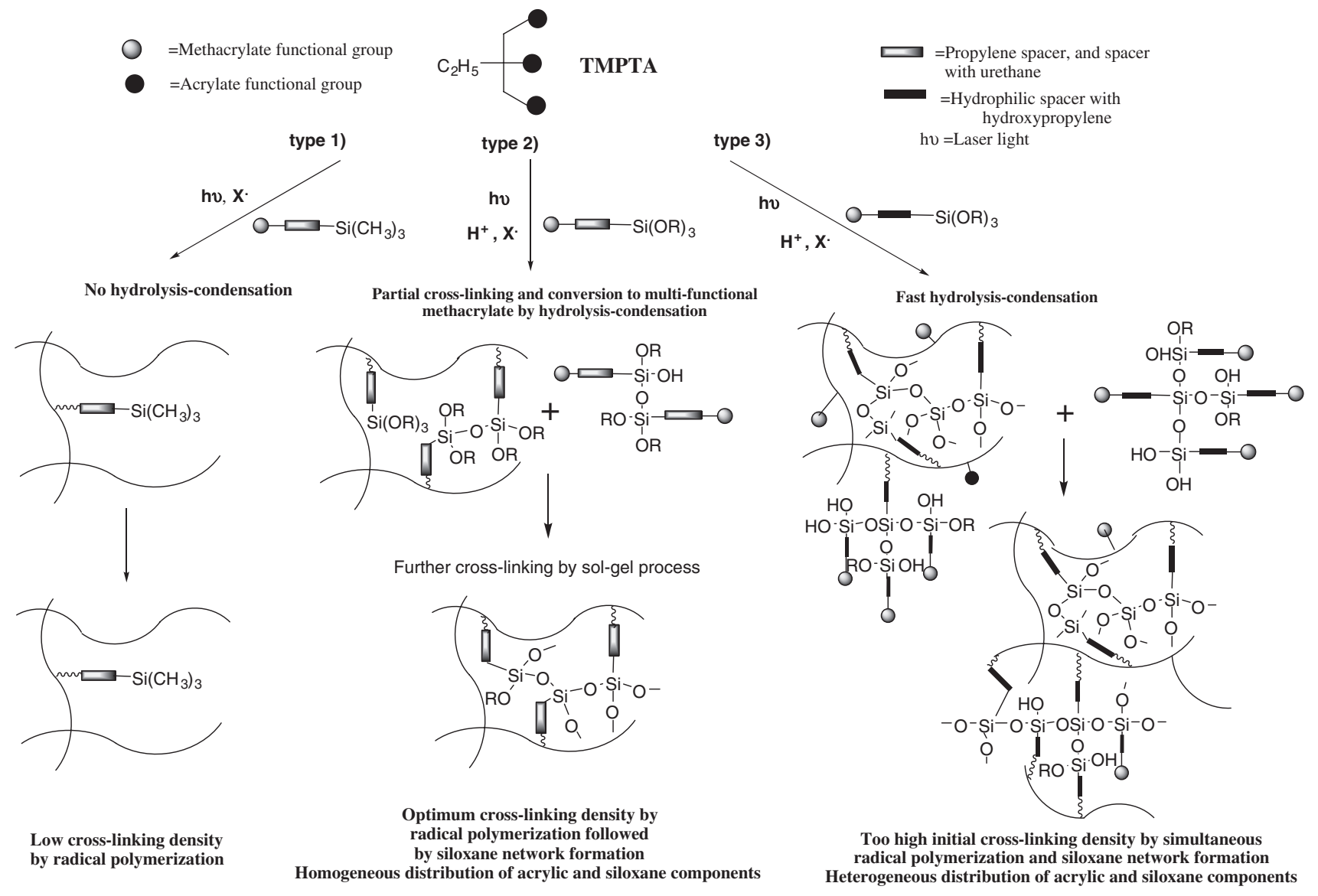

Scheme 1. Proposed matrix formation processes: 1) radical cross-linking by TMPTA, 2) simultaneous radical cross-linking of TMPTA and small amounts of multi-functional methacrylate formed via hydrolysis-condensation of trialkoxysilyl group, followed by further cross-linking by hydrolysis, 3) competing rapid cross-linking of (meth)acrylate functions and sol-gel process of trialkoxysilane function, followed by further cross-linking by radical polymerization and sol-gel process. 


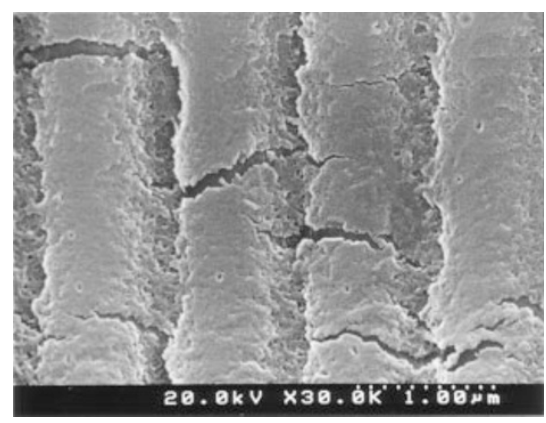

(a)

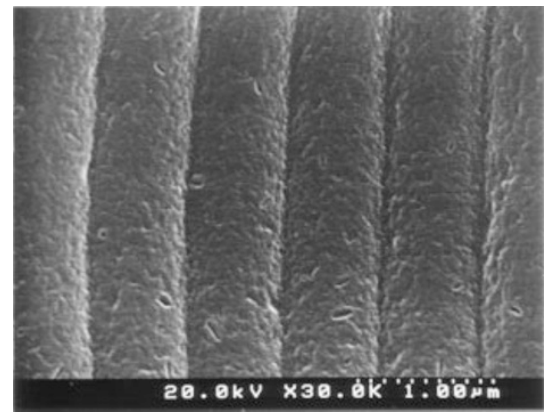

(b)

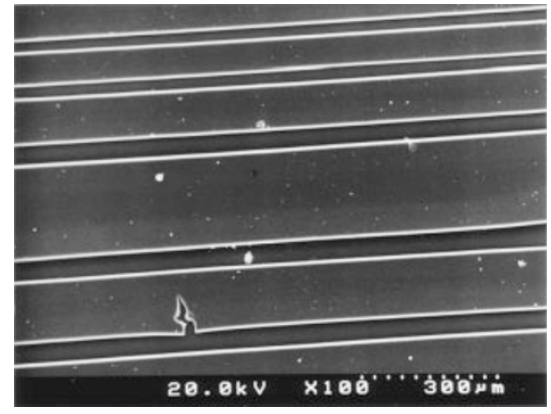

(c)

Figure 11. SEM morphologies of the gratings formed with MU-TEOS in recording solution with $65 \mathrm{wt} \%$ matrix compounds of TMPTA:MU-TEOS:NVP $=90-\mathrm{X}: \mathrm{X}: 10 \mathrm{wt} \%, \quad 35 \mathrm{wt} \%$ TL203, and KC-DPI ( $0.2 \mathrm{wt} \%-2 \mathrm{wt} \%$ to matrix compounds): $\mathrm{X}$ is (a) $80 \mathrm{wt} \%, \times 30 \mathrm{~K}$, (b) $60 \mathrm{wt} \%, \times 30 \mathrm{~K}$, and (c) $60 \mathrm{wt} \%$, $\times 100$.

Gradual formation of cross-linking, which facilitates the almost complete phase separation of LC, is very important to obtain high diffraction efficiency, and the decreased existence of few double bond is also very important for the long term stability. Well-balanced cross-linking by radical polymerization and sol-gel process in type 2) process for MU-TEOS seems to have given stable grating with high diffraction efficiency.

To study the morphology of gratings formed with MU-TEOS, SEM measurement was carried out. Figure 11 shows the surface morphologies of gratings formed with MU-TEOS at the ratio of TMPTA: MU-TEOS:NVP $=10$, or 30:80, or $60: 10 \mathrm{wt} \%$ and
TL203 (35 wt \%) after washing with methanol for over night.

Periodic gratings were clearly formed and grating spacing were about 0.9 and $0.7 \mu \mathrm{m}$ for gratings with $80 \mathrm{wt} \%$ and $60 \mathrm{wt} \%$, respectively [Figure 11(a) and (b)]. This value was in good agreement with the calculated spacing $(0.96 \mu \mathrm{m})$ by Bragg's equation. Longer grating spacing was observed in case of $80 \mathrm{wt} \%$ [Figure 11(a)] than the cases of lower concentration of $60 \mathrm{wt} \%$ MU-TEOS [Figure 11(b)]. It maybe considered that in high concentration of MU-TEOS, volume shrinkage caused during formation of polymer matrix was restrained due to higher concentration of siloxane network in polymer matrix. However, some cracks were observed in gratings formed with $80 \mathrm{wt} \%$ MU-TEOS as shown in Figure 11(a), which attributed to high vitrification of the polymer matrix, whereas, in case of $60 \mathrm{wt} \%$, there was no crack in inside of gratings even though there were some cracks in whole sample as shown in Figure 11(c).

\section{CONCLUSIONS}

Stable transmission holographic polymer dispersed liquid crystal gratings were efficiently prepared in short time via network formation by radical polymerization of tri-functional acrylate assisted by hydrolysis-condensation reaction or trialkoxysilane functional group of $\omega$-methacryloxyalkyltrialkoxysilane, induced by radical and proton species produced in the photodecomposition of initiating system composed of 3, 3'carbonylbis[7'-diethylaminocoumarine] as a photosensitizer and diphenyliodonium hexafluorophosphate as a photo-initiator.

Enhanced diffraction efficiency (86\%, induction period $144 \mathrm{~s}$ ) was observed for the grating formed with $80 \mathrm{wt} \%$ 3-methacryloxymethyltrimethoxysilane (cross-linkable by hydrolysis) with only $10 \mathrm{wt} \%$ trimethylolpropane triacrylate with $0.2 \mathrm{wt} \%$ sensitizer and $2 \mathrm{wt} \%$ initiator. Grating could not formed for non-cross-linkable 3-methacryloxymethyltrimethylsilane. The lowered performance of 3-methacryloxypropyltriethoxysilane system was improved $(75 \%$, induction period $75 \mathrm{~s}$ ) by introducing hydrophilic urethane group into the spacer between triethoxysilylpropyl and methacrylate. The longest grating spacing of $0.9 \mu \mathrm{m}$ indicated the least volume shrinkage.

Acknowledgment. This work was partly supported by a Grant-in-Aid for Scientific Research (16205016) from the Ministry of Education, Science, Sports, Culture and Technology, Japan. This work was also partly supported by a City Area Program from the Ministry of Education, Science, Sports, Culture and Technology, Japan. 


\section{REFERENCES}

1. S. Meng, K. Nanjundiah, T. Kyu, L. V. Natarajan, V. P. Tondiglia, and T. J. Bunning, Macromolecules, 37, 3792 (2004).

2. S. Meng, T. Kyu, L. V. Natarajan, V. P. Tondiglia, R. L. Sutherland, and T. J. Bunning, Macromolecules, 38, 4844 (2005).

3. T. Kyu and D. Nwabunwa, Macromolecules, 34, 9168 (2001).

4. D. J. Pikas, S. M. Kirkpatrick, D. W. Tomlin, L. Natarajan, V. Tondiglia, and T. J. Bunning, Appl. Phys. A, 74, 767 (2002).

5. R. L. Sutherland, J. Opt. Soc. Am. B, 19, 2995 (2002).

6. K. Kato, T. Hisaki, and M. Date, Jpn. J. Appl. Phys., 38, 1466 (1999).

7. T. J. White, L. V. Natarajan, V. P. Tondiglia, T. J. Bunning, and C. A. Guymon, Macromolecules, 40, 1112 (2007).

8. T. J. White, L. V. Natarajan, V. P. Tondiglia, P. F. Lloyd, T. J. Bunning, and C. A. Guymon, Macromolecules, 40, 1121 (2007).

9. K. Kato, T. Hisaki, and M. Date, Jpn. J. Appl. Phys., 38, 805 (1999).

10. L. V. Natarajan, D. P. Brown, J. M. Wofford, V. P. Tondiglia, R. L. Sutherland, P. F. Lloyd, and T. J. Bunning, Polymer, 47, 4411 (2006).

11. D. H. Choi, M. J. Cho, H. Yoon, H. Yoon, J. H. Kim, and S. H. Paek, Opt. Mater., 27, 85 (2004).

12. M. Jazbinsek, I. D. Olenik, M. Zgonik, A. K. Fontecchio, and G. P. Crawford, J. Appl. Phys., 90(8), 3831 (2001).

13. J. Zhang, C. R. Carlen, S. Palmer, and M. B. Sponsler, J. Am. Chem. Soc., 116, 7055 (1994).

14. D. E. Lucchetta, R. Karapinar, A. Manni, and F. Simoni, J. Appl. Phys., 91, 6060 (2002).

15. M. E. D. Rosa, V. P. Tondiglia, and L. V. Natarajan, J. Appl. Phys. Sci., 68, 523 (1998).

16. L. Carretero, S. Blaya, R. Mallavia, R. F. Madrigal, and A. Fimia, J. Mod. Opt., 45, 2345 (1998).

17. J. Zhang and M. B. Sponsler, J. Am. Chem. Soc., 114, 1506 (1992).

18. M. Date, Y. Takeuchi, and K. Kato, Jpn. J. Appl. Phys., 32, 3164 (1999).

19. T. J. Bunning, L. V. Natarajan, V. P. Tondiglia, R. L. Sutherland, D. L. Vezie, and W. W. Adams, Polymer, 36, 2699 (1995).

20. L. V. Natarajan, C. K. Shepherd, D. M. Brandelik, R. L. Sutherland, S. Chandra, V. P. Tondiglia, D. Tomlin, and T. J. Bunning, Chem. Mater., 15, 2477 (2003).

21. M. S. Park, B. K. Kim, and J. C. Kim, Polymer, 44(5), 1595 (2003).

22. R. L. Sutherland, L. V. Natarajan, V. P. Tondiglia, and T. J. Bunning, Chem. Mater., 5, 1533 (1993).

23. J. Zhang, C. R. Carlen, S. Palmer, and M. B. Sponsler, J. Am. Chem. Soc., 116, 7055 (1994).

24. T. J. Bunning, L. V. Natarajan, V. P. Tondiglia, and R. L. Sutherland, Annu. Rev. Mater. Sci., 30, 83 (2000).

25. Y. J. Liu, B. Zhang, Y. Jia, and K. S. Xu, Opt. Commun., 218, 27 (2003).
26. M. D. Sarkar, J. Qi, and G. P. Crawford, Polymer, 43, 7335 (2002).

27. M. J. Escuti, P. Kossyrev, and G. P. Crawford, Appl. Phys. Lett., 77, 4262 (2000).

28. D. R. Cairns, C. C. Bowley, S. Danworaphong, A. K. Fontecchio, G. P. Crawford, L. Li, and S. M. Faris, Appl. Phys. Lett., 77, 2677 (2000).

29. T. J. White, W. B. Liechty, L. V. Natarajan, V. P. Tondiglia, T. J. Bunning, and C. A. Guymon, Polymer, 47, 2289 (2006).

30. O. Soppera, C. Croutxe-Barghorn, C. Carre, and D. Blanc, Appl. Surf. Sci., 186, 91 (2002).

31. K. Saravanamuttu, C. F. Blanford, D. N. Sharp, E. R. Dedman, A. J. Turberfield, and R. C. Denning, Chem. Mater., 15, 2301 (2003).

32. J. Xu, W. Pang, and W. Shi, Thin Solid Films, 514, 69 (2006).

33. M. V. Kahraman, M. Kuğu, Y. Menceloğlu, N. Kayaman-Apohan, and A. Güngör, J. Non-Cryst. Solids, 352, 2143 (2006).

34. R. Tamaki, T. Horiguchi, and Y. Chujo, Bull. Chem. Soc. Jpn., 71, 2749 (1998).

35. J. Pyun and K. Matyjaszewski, Chem. Mater., 13, 3436 (2001).

36. P. Cheben and M. L. Calvo, Appl. Phys. Lett., 78, 1490 (2001).

37. Y. H. Cho, C. W. Shin, N. Kim, B. K. Kim, and Y. Kawakami, Chem. Mater., 17, 6263 (2005).

38. Y. H. Cho, M. He, B. K. Kim, and Y. Kawakami, Sci. Technol. Adv. Mater., 5, 319 (2004).

39. Y. H. Cho, R. Kawade, T. Kubota, and Y. Kawakami, Sci. Technol. Adv. Mater., 6, 435 (2005).

40. Y. H. Cho and Y. Kawakami, Appl. Phys. A, 83, 365 (2006).

41. Z. Li, Y. H. Cho, and Y. Kawakami, Polym. Int., 56, 666 (2007).

42. M. He, Y. H. Cho, N. Kim, and Y. Kawakami, Des. Monomers Polym., 8, 473 (2005).

43. M. He, Y. H. Cho, and Y. Kawakami, Polym. J., 38, 678 (2006).

44. M. A. Hoque, Y. H. Cho, and Y. Kawakami, React. Funct. Polym., accepted (2007).

45. Y. H. Cho and Y. Kawakami, Silicon Chem., 3, 219 (2007).

46. Z. Gomurashvili and J. V. Crivello, Macromolecules, 35, 2962 (2002).

47. Z. Gomurashvili and J. V. Crivello, J. Polym. Sci., Part A: Polym. Chem., 39, 1187 (2001).

48. J. V. Crivello and F. Jiang, Chem. Mater., 14, 4858 (2002).

49. J. V. Crivello and J. H. W. Lam, Macromolecules, 10, 1307 (1977).

50. J. V. Crivello and J. L. Lee, J. Polym. Sci., Part A: Polym. Chem., 27, 3951 (1989).

51. F. Castellanos, J. P. Fouassier, C. Priou, and J. Cavezzan, J. Appl. Polym. Sci., 60, 705 (1996).

52. M. D. Sarkar, N. L. Gill, J. B. Whitehead, and G. P. Crawford, Macromolecules, 36, 630 (2003).

53. "The Chemistry of Organic Silicon Compounds," S. Patai and Z. Rappoport, Ed., John Wiley \& Sons, Chichester, New York, Brisbane, Toronto, Singapore, 1989. 